\title{
Analytical solution for self-induced motion of a helical vortex with a Gaussian core
}

Okulov, V. L.; Fukumoto, Y.

Published in:

Thermophysics and Aeromechanics

Link to article, DOI:

$10.1134 / \mathrm{S} 0869864320040022$

Publication date:

2020

Document Version

Peer reviewed version

Link back to DTU Orbit

Citation (APA):

Okulov, V. L., \& Fukumoto, Y. (2020). Analytical solution for self-induced motion of a helical vortex with a Gaussian core. Thermophysics and Aeromechanics, 27(4), 481-488.

https://doi.org/10.1134/S0869864320040022

\section{General rights}

Copyright and moral rights for the publications made accessible in the public portal are retained by the authors and/or other copyright owners and it is a condition of accessing publications that users recognise and abide by the legal requirements associated with these rights.

- Users may download and print one copy of any publication from the public portal for the purpose of private study or research.

- You may not further distribute the material or use it for any profit-making activity or commercial gain

- You may freely distribute the URL identifying the publication in the public portal

If you believe that this document breaches copyright please contact us providing details, and we will remove access to the work immediately and investigate your claim 


\title{
Analytical solution for self-induced motion of a helical vortex with a Gaussian core*
}

\author{
V.L. Okulov ${ }^{1-3}$ and Ya. Fukumoto ${ }^{4}$
}

${ }^{1}$ Novosibirsk state University, Novosibirsk, Russia

${ }^{2}$ Kutateladze Institute of Thermophysics SB RAS, Novosibirsk, Russia

${ }^{3}$ Technical University of Denmark, Lyngby, Denmark

${ }^{4}$ Institute of Mathematics for Industry, Kyushu University, Fukuoka, Japan

E-mail: vokulov@mail.ru

(Received April 22, 2020; revised June 9, 2020;

accepted for publication July 10, 2020)

\begin{abstract}
The paper presents an analytical solution for helical vortices with a Gaussian vorticity distribution in the core, which is confirmed by experimental and numerical simulations. This result is obtained by extending the Dyson method to the Biot-Savart law. Previously, analytical solutions were found and studied only for vortices with constant vorticity distribution in the core (a Rankine-type vortex core). One of the important issues raised during the discussion is the difference between self-induced movements of helical structures with both types of vortex core. The proposed solutions are important for the fundamental understanding and description of the behavior of helical eddy flows in various fields of industry and in nature. Examples include tip vortices behind the rotors of wind or hydro turbines, tornadoes, or axial vortices in aerodynamic devices such as vortex apparatuses and generators; cyclone separators, combustion chambers, etc.
\end{abstract}

Keywords: vortex dynamics, helical vortex, Gaussian vorticity distribution, self-induced rotation.

\section{Introduction}

The dynamics of helical vortices is of fundamental importance for many energy applications, since these vortices enhance heat and mass transfer, affect the performance of rotary machines, and, conversely, their immovable, stationary position provides stable operating modes in vortex devices [1]. In recent years, the modeling of helical vortices has received a new impetus due to the study of vortex interactions in the wakes of turbines located one after the other in wind farms and hydroelectric power plants $[2,3]$. For all these applications, it is necessary to correctly predict a helical structure, describe its movement, and correctly model a helical vortex. Regular experimental study of the shape and internal structure of a helical vortex was

\footnotetext{
* In this work, V.L. Okulov received support under the contract with the Ministry of Education and Science of the Russian Federation (No. 075-15-2019-1923) and Y. Fukumoto received the grants for research from the Japan Society for the Promotion of Science (No. S17119 and No. 19K03672).
}

() V.L. Okulov and Ya. Fukumoto, 2020 
started by Professor Sergey V. Alekseenko and Sergey I. Shtork at the end of the last century. They published interesting data in article [4], including a description of the structure in the form of a Gaussian shape of the vortex core.

Earlier works on mathematical modeling of helical vortices considered a special case of a vortex core only with a constant vorticity distribution (Rankine type). The author of [5] numerically found a correlation between the velocity field induced by a helical vortex with such a finite core, and that induced by an infinitely thin helical vortex line. He combined the solution for the helical vortex line with the generalized method of approximating the Biot-Savart equations given in [6]. An additional correction term using a special $\mathrm{W}$-integral after separating out singularities in the solution for the line was later recorded and analytically confirmed in [7]. The special method of [5] used velocities induced by a helical line at finite distances from its axis calculated with infinite series of Kapteyn type [8-10], to compare it with the value obtained by another method of approximating the helical vortex in contact with a vortex ring with a similar Rankine-type vortex core. Further, approach [5] was improved by two ways of separating the singularity in the Kapteyn series [7, 11, 12], but only for the case of uniform vorticity distribution in the core. However, the basic experiment [1] and a few recent experimental studies $[13,14]$ and numerical calculations $[15,16]$ unambiguously predict only the Gaussian vorticity distribution in the helix core. Due to the complexity of equations governing the induced motion of helical vortices, it has not yet been possible to obtain a solution describing motion with a Gaussian core. This result can be achieved by generalizing the Dyson decomposition for the Biot-Savart law, which induces a velocity field for helical vortices with any vorticity distribution in the final core, which is replaced on the helical axis by a sum of singularities of different degrees: a monopole, a dipole, etc. An approximation of the induced velocity for both helical vortices with Rankine and Gauss vorticity distributions in the final core was proposed by the authors of $[17,18]$ with some restriction on these approximations from the monopole and dipole. It should be noted that both solutions in $[17,18]$ were not used to estimate the self-induced motion of a helical vortex, but they will be presented below in this article. A significant result was obtained when comparing data calculated using the scheme of [5] and a new algorithm based on solutions of [17] for the Rankine core in order to establish their correlation, and the next result should be the determination of the self-induced motion of a helical vortex with a Gaussian vortex core.

This The article is organized as follows. Sections 1 and 2 recall the approximation for Kapteyn series with the addition of some terms and describe the velocity field induced by the monopole and dipole helical lines corrected by new terms in both approximations. Section 3 describes the Dyson method. In sections 4 and 5, the ideal correlation of two solutions for a helical vortex with a finite Rankine-type core is discussed, and the self-induction rate motion for a vortex with a Gaussian core is verified by solving the Navier-Stokes equations proposed in the study [16]. Section 6 is the Conclusion.

\section{Approximation of Kapteyn series}

The Kapteyn series included in the Kawada-Hardin solution are used as the basis for determining the movement of monopole and dipole helical lines [8-10]. These series can be written as [11]

$$
H_{M}^{I, J}(a, b, \chi)=\sum_{m=1}^{\infty} m^{M} I_{m}^{\langle I\rangle}(m a) K_{m}^{\langle J\rangle}(m b) \cdot e^{i m \chi},
$$

where $I_{m}^{\langle 0\rangle}(m a)$ and $K_{m}^{\langle 0\rangle}(m b)$ are the modified Bessel functions and their corresponding derivatives. In [11], an exact analytical approximation of the Kapteyn series (1), including five main terms and a small remainder that can be ignored, was derived. To solve this problem, we 
78

79

80

recall such efficient procedure for calculating Kapteyn series for a monopole line [12] in order to generalize it to the case of a dipole:

$$
\begin{aligned}
H_{M}^{I, J}(a, b, \chi)=\lambda^{I, J}\left[B_{M, 0}^{I, J}\right. & \frac{e^{\xi+i \chi}}{\left(e^{\xi}-e^{i \chi}\right)^{2}}+B_{M, 1}^{I, J} \frac{e^{i \chi}}{e^{\xi}-e^{i \chi}}+B_{M, 2}^{I, J} \ln \left(1-e^{-\xi+i \chi}\right)+ \\
& \left.+B_{M, 3}^{I, J} \mathrm{Li}_{2}\left(e^{-\xi+i \chi}\right)+B_{M, 4}^{I, J} \mathrm{Li}_{3}\left(e^{-\xi+i \chi}\right)\right]+R_{M}^{I, J}(a, b, \chi)
\end{aligned}
$$

81

82

$$
\text { where } \operatorname{Li}_{k}(z)=\sum_{m=1}^{\infty} \frac{z^{m}}{m^{k}}, \quad|z|<1 \text { are polylogarithms, } e^{\xi}=\frac{a}{b} \frac{\exp \left(\sqrt{1+a^{2}}\right)\left(1+\sqrt{1+b^{2}}\right)}{\left(1+\sqrt{1+a^{2}}\right) \exp \left(\sqrt{1+b^{2}}\right)},
$$

$$
\lambda^{I, J}=\frac{1}{2} \frac{\left(\sqrt{1+a^{2}}\right)^{I-\frac{1}{2}}\left(\sqrt{1+b^{2}}\right)^{J-\frac{1}{2}}}{a^{I}(-b)^{J}} \text { and } \mathbf{B}^{I, J}=\left[\begin{array}{cccccc}
0 & 0 & 1 & \alpha^{I, J} & \beta^{I, J} & \gamma^{I, J} \\
0 & 1 & \alpha^{I, J} & \beta^{I, J} & \gamma^{I, J} & 0 \\
1 & \alpha^{I, J} & \beta^{I, J} & \gamma^{I, J} & 0 & 0
\end{array}\right] \text {. }
$$

The matrix components $\mathbf{B}^{I, J}$ that are used to calculate the velocity field over the entire space are defined as

86

87

$$
\begin{gathered}
\alpha^{I, J}(x, y)=(1-I) \theta_{1}(x)-(1-J) \theta_{1}(y)+I v_{1}(x)-J v_{1}(y) \\
\beta^{I, J}(x, y)=(1-I) \theta_{2}(x)+(1-J) \theta_{2}(y)+I v_{2}(x)+J v_{2}(y)- \\
-(1-I)(1-J) \theta_{1}(x) \theta_{1}(y)-J(1-I) \theta_{1}(x) v_{1}(y)-I(1-J) \theta_{1}(y) v_{1}(x)-I J v_{1}(x) v_{1}(y), \\
\gamma^{I, J}(x, y)=(1-I) \theta_{3}(x)-(1-J) \theta_{3}(y)+I v_{3}(x)-J v_{3}(y)+ \\
+(1-I)(1-J)\left[\theta_{1}(x) \theta_{2}(y)-\theta_{2}(x) \theta_{1}(y)\right]+J(1-I)\left[\theta_{1}(x) v_{2}(y)-\theta_{2}(x) v_{1}(y)\right]- \\
+I(1-J)\left[\theta_{2}(y) v_{1}(x)-\theta_{1}(y) v_{2}(x)\right]+I J\left[v_{1}(x) v_{2}(y)-v_{2}(x) v_{1}(y)\right] .
\end{gathered}
$$

88

The functions included in the definitions of $\alpha^{I, J}, \beta^{I, J}, \gamma^{I, J}$, are polynomials in

$$
t \equiv x=\left(1+a^{2}\right)^{-1 / 2} \text { or } t \equiv y=\left(1+b^{2}\right)^{-1 / 2}: \quad \theta_{1}=\left(3 t-5 t^{3}\right) / 24, \quad v_{1}=\left(-9 t+7 t^{3}\right) / 24 \text {; }
$$

$$
\theta_{2}=\left(81 t^{2}-462 t^{4}+385^{6}\right) / 1152, \quad v_{2}=\left(-135 t^{2}+594 t^{4}-455 t^{6}\right) / 1152
$$

$$
\begin{gathered}
\theta_{3}=\left(30375 t^{3}-369603 t^{5}+765765 t^{7}-425425 t^{9}\right) / 414720, \\
v_{3}=\left(-42525 t^{3}+451737 t^{5}-883575 t^{7}+475475 t^{9}\right) / 414720 .
\end{gathered}
$$

The small regular remainder in (2) is defined by the term

94

$$
\begin{aligned}
R_{M}^{I, J} & =\sum_{m=1}^{\infty} r i_{m}(a, b, I, J, M) \cdot e^{i m \chi}= \\
& =\sum_{m=1}^{\infty}\left[m^{M} I_{m}^{\langle I\rangle}(m a) K_{m}^{\langle J\rangle}(m b)-m^{M} \lambda^{I, J}\left(e^{\xi}\right)^{m}\left(1+\frac{\alpha^{I, J}}{m}+\frac{\beta^{I, J}}{m^{2}}+\frac{\gamma^{I, J}}{m^{3}}\right)\right] e^{i m \chi} .
\end{aligned}
$$


Obtaining accurate results for calculating flows using a series (1) in the azimuthal direction comes amid errors due to the approximation of an infinite series by a finite set of harmonics. In this paper, to correctly model the velocity field, we use the approximation (2), in which the residual term (3) is small, and it can be ignored in formula (2) based on the data in Table 1 and Fig. 5 from [11].

\section{The velocity field induced by monopole and dipole helical lines}

Let us start by defining the geometry of a helical line of radius $R$ in accordance with Fig. $1 a$ from article [19]. Helical pitch $L=2 \pi l$ is defined as the displacement along the axis at one revolution of a helix, and the helical angle is determined by the ratio $\tan \phi=L / 2 \pi R$ with the corresponding torsion of the helix $\tau=l / R$. In addition, the absolute velocity $U$ induced by a helical vortex with a finite core radius $\varepsilon=\sigma R$ is determined either through binormal $U_{\mathrm{b}}$ and tangential $U_{\mathrm{t}}$ components, or through the axial $U_{z}$ and azimuthal $U_{\theta}$ velocity projections. In the context of these same components of the total velocity $U$, the components of velocities induced by the monopole or dipole distributing along helical lines will be defined, but using the symbols $u$ and $v$, respectively.

In cylindrical coordinates $(r, \theta, z)$, according to the Kawada-Hardin solution [8-10], the axial $u_{z}$ and azimuthal $u_{\theta}$ components of velocity induced by a monopole helical line outside the singular core have the form

$$
u_{\theta}(r, R, \chi)=\frac{\Gamma}{2 \pi r}\left\{\begin{array}{l}
0 \\
1
\end{array}\right\}+\frac{\Gamma a}{\pi r l}\left\{\begin{array}{l}
H_{1}^{0,1}(r / l, R / l, \chi) \\
H_{1}^{1,0}(R / l, r / l, \chi)
\end{array}\right\}, \quad u_{z}(r, R, \chi)=\frac{\Gamma}{2 \pi l}-\frac{r}{l} u_{\theta}(r, R, \chi),
$$

where $\chi=\theta-z / l$, the upper expression in curly brackets corresponds to the case $r<R$, and the lower expression corresponds to $r>R$.

The first representation of the velocity field induced by a dipole helical line was obtained using an exact analytical approximation through Kapteyn series in [17, 18]:

$$
v_{\theta}=4 d \frac{R^{2}+l^{2}}{r R l^{2}} \operatorname{Re}\left\{\begin{array}{c}
H_{2}^{0,0}(r / l, R / l, \chi) \\
H_{2}^{0,0}(R / l, r / l, \chi)
\end{array}\right\}, \quad v_{z}=-4 d \frac{R^{2}+l^{2}}{R l^{3}} \operatorname{Re}\left\{\begin{array}{l}
H_{2}^{0,0}(r / l, R / l, \chi) \\
H_{2}^{0,0}(R / l, r / l, \chi)
\end{array}\right\},
$$

where $d$ is the intensity of the dipole moment with the binormal direction of the dipole moment vector to the helical line.

\section{Dyson's method for approximating a helical vortex with a finite core}

In accordance with the procedure described in article [17], we will explain the main points of the Dyson method, which will be used to derive the final equations that determine the components of the total velocity $U$. In [20] to study Saturn rings, Dyson proposed an original high-precision method for estimating the velocity field in the vicinity of the core of an axisymmetric vortex ring with a vorticity distribution proportional to the distance from the axis of the ring. The use of the shift operator and the properties of harmonic functions for the velocity field near the core allowed obtaining effective high-order asymptotic expansions that depend on a small parameter, the ratio of radii of the core and the ring. This method was generalized to an axisymmetric vortex ring with an arbitrary vorticity distribution, and the formula for selfinduced motion of a viscous vortex ring was improved for higher orders [21]. In fact, the Dyson method is extremely important for achieving high-order decompositions, since otherwise 
decompositions may encounter a number of uncertain conditions. Later, the restriction of axial symmetry was removed for the Dyson method [17], whereby, for a helical vortex tube, the influence of its finite core was successfully represented as multipole decompositions. Here, the small parameter $\sigma$ is the ratio of the core radius to the normal radius of curvature. In this paper, shift operators were first used to perform integration across the core cross section in a volume integral, which then led it to a linear integral. The main term of the decomposition of the Biot-Savart integral was represented by a chain of monopole singularities located on the central line of a helical vortex tube. The intensity of monopoles is equal to the circulation of a helical vortex. The next contribution was made by a chain of dipoles whose intensity depends on the vorticity distribution in the core. The key factor for dipoles was the influence of the curvature and torsion of the helix. Similar to the case of monopoles (4), the velocity field for a helical dipole line is written as Kapteyn type series (5). In this study, based on estimates of [17], we consider only the contribution of these two singularities:

$$
U_{z}=u_{z}+v_{z}, \quad U_{\theta}=u_{\theta}+v_{\theta}, \quad U_{\mathrm{b}}=u_{\mathrm{b}}+v_{\mathrm{b}}, \quad U_{\mathrm{t}}=u_{\mathrm{t}}+v_{\mathrm{t}},
$$

where the binormal and tangential velocity components correlate accordingly with the azimuthal and axial components $u_{\theta}$ and $u_{z}$ or $v_{\theta}$ and $v_{z}$ through obvious relations $[5,12]$ :

$$
u_{\mathrm{b}}=\frac{\tau u_{\theta}-u_{z}}{\sqrt{1+\tau^{2}}}, \quad u_{\mathrm{t}}=\frac{u_{\theta}+\tau u_{z}}{\sqrt{1+\tau^{2}}} \quad \text { and } \quad v_{\mathrm{b}}=\frac{\tau v_{\theta}-v_{z}}{\sqrt{1+\tau^{2}}}, v_{\mathrm{t}}=\frac{v_{\theta}+\tau v_{z}}{\sqrt{1+\tau^{2}}}
$$

It should be noted that the relative displacement of the vortex is determined only by the values $u_{\mathrm{b}}$ and $v_{\mathrm{b}}$, while $u_{\mathrm{t}}$ gives a purely tangential motion along the axis, and with the help of substitution (5) in $v_{\mathrm{t}}=v_{\mathrm{z}}+r v_{\theta} / l$ we find that $v_{\mathrm{t}}$ is zero. In accordance with section 2, sufficient accuracy for infinite summation of the main components of velocities $u$ and $v$ in (6) is achieved when singularities are separated out using the closed analytical form (2).

\section{Comparison of two calculation methods for a vortex with a Rankine core}

The next question of interest concerns the verification of the Dyson method for describing the self-induced motion of a helical vortex with a finite core size of constant vorticity through representations of the velocity of monopole and dipole singular vortex lines (6). A simple way to estimate the velocity in a vortex is to take the value $U$ between two diametrically opposite points on the radius of the vortex core $R=\varepsilon / \sigma$, that is, at the point $(r, \theta, z)=(R \pm \varepsilon, 0,0)$, then the motion of the vortex will be represented by the average of these two values:

$$
U_{\mathrm{b}_{\text {SInd }}}=\frac{1}{2}\left[U_{\mathrm{b}}(R-\varepsilon)+U_{\mathrm{b}}(R+\varepsilon)\right] \equiv \frac{1}{2}\left[u_{\mathrm{b}}(R-\varepsilon)+v_{\mathrm{b}}(R-\varepsilon)+u_{\mathrm{b}}(R+\varepsilon)+v_{\mathrm{b}}(R+\varepsilon)\right] .
$$

Previously, this procedure was used for a monopole helical line only with $u_{\mathrm{b}}$, but such an analysis performed in [5, 7, and 11] requires an additional correction for the finite core found by direct comparison with the solution for the corresponding vortex ring [6]. The first realistic formula for the binormal velocity of a helical vortex with a finite core

$$
U_{\mathrm{b}_{B \& W}}=\frac{1}{2}\left[u_{\mathrm{b}}(R-\varepsilon)+u_{\mathrm{b}}(R+\varepsilon)\right]+\Delta_{\varepsilon} \text { was obtained in [7]: }
$$

$$
\frac{4 \pi R}{\Gamma} U_{\mathrm{b}_{B \& W}}=\frac{1}{1+\tau^{2}}\left[\ln \left(\frac{2}{\sigma}\right)-\frac{1}{4}+2 \tau^{2}-2 \tau \sqrt{1+\tau^{2}}-\ln \sqrt{1+\tau^{2}}+\left(1+\tau^{2}\right)^{3 / 2} W(\tau)\right]
$$




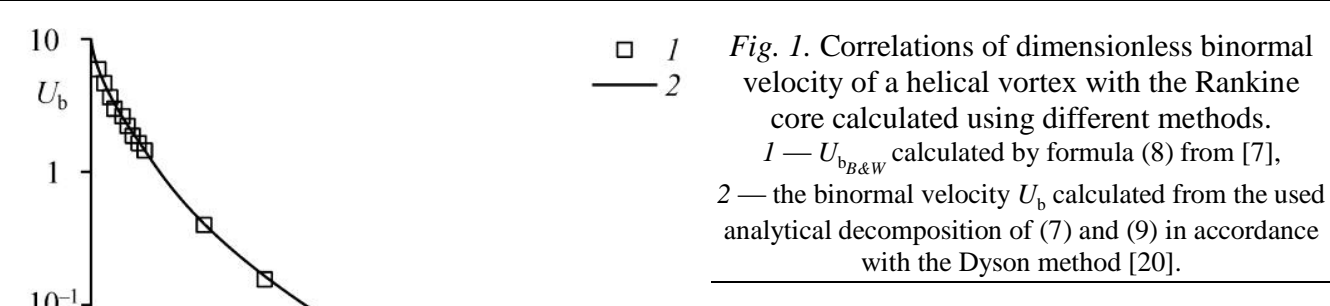

where the additional term " $1 / 4$ " in square brackets defines the correction $\Delta_{\varepsilon}$, associated with the finite core of the helical vortex, and

$$
W(\tau)=\int_{0}^{\infty}\left\{\frac{\sin ^{2} t}{\left[\tau^{2} t^{2}+\sin ^{2} t\right]^{3 / 2}}-\frac{1}{\left[\tau^{2}+1\right]^{3 / 2}} \frac{H(1 / 2-t)}{t}\right\} d t
$$

describes the regular part of Kapteyn series with subtraction of the pole $1 / \sigma$ and the logarithm $\ln (1 / \sigma)$ from the original representation of the series (1), where $H()$ denotes a the step function. However, for helical vortex lines, equation (8), as well as the Biot-Savart law, cannot be integrated in a simple analytical form, so $W$ was found numerically with an accuracy of up to six significant digits for the 21 st step values of $\tau$, which is shown in the table of [7]. As before, the analytical form of the Kapteyn series (2) in combination with formulas (4) and (5) after substitution in (7) will be is used to obtain analytical expressions for self-induced velocities. To compare the Dyson method (7) with the formula (8), we need the value of the dipole moment intensity for the Rankine vortex in the core of the vortex. The estimate from [17] gives the formula

$$
d_{R}=-3 \Gamma \sigma^{2} / 16 \pi
$$

The binormal velocity (see 2 in Fig. 1) is calculated as a function of the torsion using the new analytical formula (7). It is compared with solution 1 of [7], where the $\mathrm{W}$-integral formula (8) was used. The results obtained by both methods demonstrate excellent consistency, which proves the high accuracy of the Dyson method for determining the self-induced velocity of helical vortices with a Rankine core. Additionally, the approximation (2) of Kapteyn series for velocity fields induced by monopole and dipole lines was performed.

\section{Self-induced velocity for a helical vortex with a Gaussian core}

To obtain the self-induced velocity of a helical vortex, we use the Dyson approach (7) to estimate the velocity field induced in the vicinity with a non-constant Gaussian vorticity distribution in the core. In contrast to the Rankine vortex (9), the intensity of the dipole moment for the Gaussian vorticity distribution takes a different value. Comparison with experimental data [16] gives the formula

$$
d_{\mathrm{G}}=-0.13 \text {. }
$$

In Fig. 2, the self-induced angular velocity calculated via the binormal component (7) for a Gaussian vortex core (line 2) with dipole intensity (10) gives a good agreement with the data of direct numerical simulation (DNS) based on the solution of the Navier-Stokes equations for 
Fig. 2. Angular velocity of helical vortex $\Omega_{\text {Sind }}=\left(-U_{\mathrm{b}} / \sin \phi\right) / R[11]$ with a pitch $l=0.5$, as a function of the vortex core radius $\sigma$ with vorticity distribution of Gaussian type. 1 - numerical simulation using DNS method [16] 2 - calculation using formula (7) with dipole intensity (10),

3 - inaccurate data for the first two terms from (2) at $d=0.103$ from [17],

4 - angular velocity of a vortex with a Rankine core calculated by (7) and (9) of this work or by (8) from [7]. in time with a fixed pitch of $l=0.5$ (see Fig. 9b from [16]). Thus, for the first time

\section{Conclusion}

\section{References} Aeromechanics, 1996, Vol. 3, No. 2, P. 97-128. Series, 2014, Vol. 524, No. 1, P. 012162. Russia, P. 134. Thermophys., 1992, Vol. 2, No. 4, P. 231-266. P. $241-259$. 1972, Vol. 272, P. 403-429. 279. P. 060801. 2015, Vol. 780, P. 687-716.

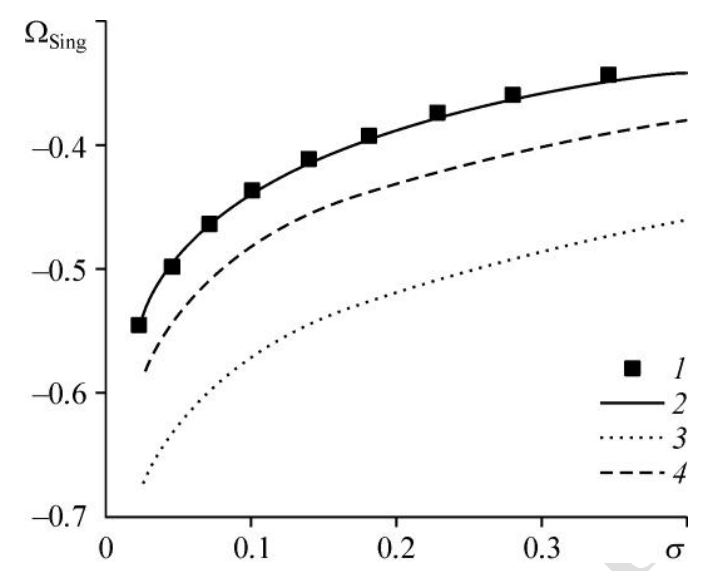
in a closed form, solution (7) with dipole intensity (10) is obtained to describe the motion of a helical vortex in space with a Gaussian vorticity distribution in the core.

In this paper, we have tested the Dyson method for determining the velocity field in the vicinity of helical vortices with a Rankine core by direct comparison with another estimate of the self-induced velocity obtained using Ricca modeling [1], which has been analytically proved by the authors [3]. The high accuracy of the Dyson method is demonstrated, which opens the possibility for developing new fundamental solutions, in particular, for a helical vortex with a Gaussian core. The proposed solutions can be used to describe various typical vorticity distributions in the vortex core, which give a more realistic description in applications, for example, for tip vortices behind rotors, tornadoes or axial vortices in aerodynamic devices such as vortex apparatuses and generators: cyclone separators and combustion chambers, etc.

1. S.V. Alekseenko and V.L. Okulov, Swirl flows in technical applications (A Review), Thermophysics and

2. N.G. Nygaard, Wakes in very large wind farms and the effect of neighbouring wind farms, J. Physics: Conference

3. V.L. Okulov, I.K. Kabardin, I.V. Litvinov, R.F. Mikkelsen, I.V. Naumov, J.N. Sørensen, D.H. Wood, and S.V. Alekseenko, Hydrokinetic energy conversion: the basis of hydro farm, in: Abs. 5th International Workshop on Heat/Mass Transfer Advances for Energy Conservation and Pollution Control August 13-16 2019, Novosibirsk,

4. S.V. Alekseenko and S.I. Shtork, Swirling flow large-scale structures in a combustor model, Russ. J. Engng

5. R.L. Ricca, The effect of torsion on the motion of a helical vortex filament, J. Fluid Mech., 1994, Vol. 273,

6. D.W. Moore and P.G. Saffman, The motion of a vortex filament with axial flow, Phil. Trans. R. Soc.Lond. A,

7. J. Boersma and D.H. Wood, On the self-induced motion of a helical vortex, J. Fluid Mech., 1999, Vol. 384, P. 263

8. S. Kawada, Induced velocity by helical vortices, J. Aeronaut. Sci., 1936, Vol. 3, No. 3, P. 86-87.

9. J.C. Hardin, The velocity field induced by a helical vortex filament, Phys. Fluids, 1982, Vol. 25, P. 1949-1952.

10. Y. Fukumoto, V.L. Okulov, and D.H. Wood, The contribution of Kawada to the analytical solution for the velocity induced by a helical vortex filament, ASME. Appl. Mech. Rev., 2015, Vol. 67, No. 6,

11. V.L. Okulov, On the stability of multiple helical vortices, J. Fluid Mech., 2004, Vol. 521, P. 319-342.

12. V.L. Okulov and J.N. Sørensen, The self-induced motion of a helical vortex, J. Fluid Mech., 2020, Vol. 883, A-5.

13. H.U. Quaranta, H. Bolnot, and T. Leweke, Long-wave instability of a helical vortex, J. Fluid Mech.,

14. V.L. Okulov, I.K. Kabardin, R.F. Mikkelsen, I.V. Naumov, and J.N. Sørensen, Helical selfsimilarity of tip vortex cores, J. Fluid Mech., 2019, Vol. 859, P. 1084-1097. 
15. M. Ali and M. Abid, Self-similar behavior of a rotor wake vortex core, J. Fluid Mech., 2014, Vol. 740, R1.

16. C. Selçu, I. Delbende, and M. Rossi, Helical vortices: quasiequilibrium states and their time evolution, Phys. Rev. Fluids, 2017, Vol. 2, P. 084701.

17. Y. Fukumoto and V.L. Okulov, The velocity field induced by a helical vortex tube, Phys. Fluids, 2005 Vol. 7, No. 10, P. 107101.

18. V.L. Okulov and Ya. Fukumoto, Helical dipole, Doklady Physics, 2004, Vol. 49, No. 11, P. $662-667$.

19. V.L. Okulov, E.S. Gesheva, P.A. Kuibin, S.I. Shtork, J. Sørensen, D. Wood, and S.V. Alekseenko, Difference in the movement of helical vortex and particles along its axis, Thermophysics and Aeromechanics, 2020, Vol. 27, No. 4. P. 473-480 499-506

20. F.W. Dyson, The potential of an anchor ring. II, Philos. Trans. R. Soc. London, Ser. A, 1893, Vol. 184, P. 1041-1106.

21. Y. Fukumoto and H.K. Moffatt, Motion and expansion of a viscous vortex ring, J. Fluid Mech., 2000, Vol. 417, P. 1-45. 